\section{División sexual del trabajo en los call centers en Chile: Apropiación de habilidades "femeninas"}

Sexual division of labor in call centers in Chile: Appropriation of "feminine" skills

\section{Areli Escobar-Salazar}

\section{Resumen}

Este artículo pretende aportar al debate sobre la división sexual del trabajo en base a un estudio empírico sobre los/as trabajadores/as de call centers en Chile. Los resultados de la investigación se discuten con aportes de la teoría feminista y de la corriente operaista italiana, y proponen la apropiación de habilidades y capacidades humanas subjetivas "femeninas" formadas en los procesos de socialización, el debilitamiento de las distinciones entre el mundo dentro/fuera del trabajo, y la explotación ampliada del trabajo reproductivo de las mujeres trabajadoras y de su red familiar.

Programa de Doctorado en Investigación Social y Cultural, Universidad Autónoma de Barcelona, España. Correo electrónico: areli21@gmail.com
Palabras clave: división sexual del trabajo, habilidades subjetivas, trabajo reproductivo, call centers.

\section{Abstract}

This article seeks to contribute to the debate on sexual division of labor, based on an empirical study about workers of call centers in Santiago, Chile. The research findings are discussed in light of feminist theory and Italian operaismo, suggesting the appropriation of skills and subjective human capacities, formed through the socialization process and considered as "feminine", the weakening of the distinctions between the worlds inside/outside the workplace, and the extended exploitation of reproductive labor of women workers and that of their family network.

Key words: sexual division of labor, subjective skills, reproductive work, call centers.

\section{Introducción}

La división sexual del trabajo es de relevancia clave en cuanto permite analizar las relaciones sociales de género en el mundo laboral y las conexiones del ámbito laboral con el doméstico. Aunque los debates sobre la división sexual del trabajo forman parte de las discusiones feministas desde hace décadas, el nuevo escenario del capitalismo actual y las dinámicas del mercado laboral imponen nuevos desafíos analíticos.

Las reflexiones sobre la división sexual del trabajo se realizan en base a una investigación empírica más amplia que se orientó a explorar la subjetividad laboral de los/as trabajadores/ 
as de los call centers en Chile. Una categoría central para entender las nuevas formas de organización del trabajo en el capitalismo son las relaciones sociales de género, y la división sexual del trabajo como ámbitos necesarios de tener en cuenta, simultáneamente, en el análisis de las realidades sociales estudiadas.

Los call centers subcontratistas o fábricas de la charla ${ }^{1}$ inician sus actividades a fines de la década de los 90 en Chile, como parte de las dinámicas de deslocalización de los capitales extranjeros en busca de menores costos de producción $\mathrm{y}$, en especial, del trabajo. La revolución científico-tecnológica e informática, la masificación de los "trabajos digitales" y de los servicios empresariales a distancia en el escenario de un rápido movimiento de capitales a nivel mundial son fenómenos que también definen la emergencia de las fábricas de la charla en países de América Latina como Argentina, Brasil y México y, posteriormente, en Chile.

Los datos disponibles indican que la gran mayoría de los call centers subcontratistas presentes en Chile son grandes empresas que prestan sus servicios fundamentalmente a empresas del sector financiero y de telecomunicaciones. La mitad de ellas corresponde a capitales multinacionales. Al igual que en los otros países latinoamericanos (Del Bono 2010; Micheli 2007; Mazzei 2006; Colectivo Situaciones 2006), la industria se ha caracterizado por un rápido

\footnotetext{
La centralidad del lenguaje en los call centers motiva a Paolo Virno a denominarlos fábricas de la charla. "En la red con la que trabajo en Italia llamamos a los call centers "fábricas de la charla". Se trata del núcleo central del posfordismo: el lenguaje puesto a trabajar, la identidad entre trabajo y comunicación verbal. El lenguaje incluido en el trabajo es una novedad absoluta, la novedad que marca nuestra época" (Cit. Colectivo Situaciones 2006: 147)
}

crecimiento y un explosivo aumento de la fuerza de trabajo compuesta mayoritariamente por teleoperadores/as que ocupan la amplia base piramidal de la estructura organizativa de estas empresas².

A comienzos de la presente década muchos call centers que operaban en el país centralizaron sus plataformas de atención telefónica en otros países de América Latina como Perú y Colombia, con menores precios en los salarios y una mayor flexibilidad laboral, lo que ha generado reducciones en las plantas de estas empresas ${ }^{3}$. Los estudios realizados en Chile (Centro de Estudios Nacionales de Desarrollo Alternativo y Fundación Instituto de Estudios Laborales 2010; Molina y Ruminot 2010; Uribe y Morales 2010; Kremerman 2005) demuestran que, al igual que en otros países latinoamericanos, la fuerza de trabajo utilizada en los call centers es fundamentalmente femenina y, particularmente, de mujeres jóvenes.

Por ello, el rápido crecimiento de la industria, su magnitud, su posición en el escenario global, el tipo de habilidades requeridas, entre otros factores, hace que las relaciones sociales de género sean un criterio analítico fundamental para el estudio de las fábricas de la charla. De allí que éste sea el foco del artículo propuesto.

La fuerza de trabajo ocupada en los call centers en Chile ha aumentado rápidamente, desde 13.300 posiciones de trabajo en el año 2008 a 26.000 en el año 2009, y desde 15 mil trabajadores/ as ocupados/as a 28 mil en el mismo periodo (Benchmarking Acec 2008-2009 Cit. en Uribe y Morales 2010).

3 Según la Asociación de Empresarios de Call Centers de Chile -ACEC- (en entrevista realizada para el estudio), emplear a un operador de call center en Perú es $25 \%$ más barato que en Chile, y en Colombia un $17 \%$. El movimiento de las empresas ha generado reducciones en la fuerza de trabajo de los call centers, como se observa en el caso de Atento que redujo su planta de 7.800 personas en el año 2008 a 5.000 en el 2010 (Economía y Negocios, 8 de abril de 2010). 


\section{Cuestiones metodológicas}

El procedimiento metodológico general que se utilizó en este estudio consideró dos enfoques centrales. Por un lado, se adoptó la vinculación de lo general y lo particular, enfatizando en la relación entre el contexto global de organización del trabajo y la perspectiva de los propios sujetos respecto a su trabajo. Por otro lado, se adoptó un enfoque histórico, que permitió comprender la industria en el marco de las dinámicas económico-sociales de las últimas décadas en América Latina y Chile.

Para el estudio de los aspectos materiales de la organización del trabajo en los call centers y su inserción en el contexto global se utilizó el análisis de documentos y la entrevista a expertos/as. Se entrevistó a doce de ellos/as: ocho investigadores/as del mundo del trabajo vinculados con la temática, cuatro dirigentes/ as de organizaciones sindicales del sector, y dos representantes de las empresas.

Para conocer la perspectiva de los/as trabajadores/as de call centers se utilizaron las entrevistas en profundidad, las cuales permitieron aproximarse a la visión de los sujetos tal como la expresan en sus propias palabras (Taylor y Bogdan 1986). Se elaboró un diseño cualitativo con una muestra no probabilística, intencionada y diversificada que consideró la concentración del $80 \%$ de los/as trabajadores/ as en la capital del país (Kremerman 2005), y la participación de un $70 \%$ de mujeres en la fuerza de trabajo del sector (Uribe y Morales 2010).

En base a estos criterios se entrevistó a cuarenta trabajadores/as de doce empresas de call centers en Santiago de Chile: treinta y dos teleoperadores/as y ocho supervisores/ as. Se entrevistó a veinticinco mujeres y siete hombres con edades entre los veintidós y los treinta y cinco años, con un promedio de veintiocho años. Para el desarrollo de las entrevistas en profundidad se utilizó como instrumento de recopilación de datos una pauta semiestructurada con cinco temas centrales: trayectoria laboral, organización del trabajo, condiciones laborales, trabajo doméstico y datos personales.

La tercera técnica utilizada fue la observación directa en tres grandes empresas de call centers: Atento, Multivoice y Teleperformance. El uso combinado de las diversas técnicas de recopilación de datos logró aportar información variada y complementaria que permitió una aproximación más densa al objeto de estudio propuesto.

Se utilizaron diversos tipos de registro y análisis de la información obtenida. Para el tratamiento de los datos textuales se realizó la transcripción como corpus inicial, el que fue revisado en varias oportunidades para elaborar la malla temática, entendida como un instrumento analítico orientado a "capturar" significados contenidos en el discurso. Con la malla temática se definieron temas y subtemas relevantes para el estudio, y se utilizó la estrategia de análisis temático orientada a la captura de toda la variabilidad de posicionamientos posibles en cada contenido investigado (Baeza 2002).

Los datos obtenidos en la observación directa se registraron en un diario de campo donde se anotaron las observaciones del entorno de trabajo, los esquemas de distribución física, y los relacionamientos y comentarios de la investigadora. El análisis final de los datos consideró la combinación analítica de 
los resultados de las diversas técnicas de recopilación de información utilizadas.

\section{División sexual del trabajo: Breves apuntes conceptuales}

Para analizar la desigualdad entre hombres y mujeres es necesario considerar las relaciones sociales de clase y de género, que no son jerarquizadas sino más bien coextensivas. Se trata de conceptos que se sobreponen parcialmente, y no que se "recortan" o se "articulan" (Kergoat Cit. en Hirata 2002).

La división sexual del trabajo es parte integrante de la división social del trabajo, y hace referencia a la relación social hombres/mujeres, que atraviesa y es atravesada por otras modalidades de división social del trabajo (Kergoat Cit. en Hirata 2002). Desde un punto de vista histórico, es posible observar que la estructura actual de la división sexual del trabajo surgió simultáneamente al capitalismo, y que la relación asalariada no habría podido establecerse en ausencia del trabajo doméstico. Desde el nacimiento del capitalismo al período actual, las modalidades de esa división del trabajo entre los sexos, tanto el trabajo asalariado como el trabajo doméstico, evolucionaron en el tiempo de manera concomitante a las relaciones de producción.

En el capitalismo contemporáneo, la división sexual del trabajo permite evidenciar que los roles de género atribuidos socialmente confieren ciertas cualidades que son reconocidas como propias de la fuerza de trabajo femenina o masculina (Benería y Roldán 1992). En este sentido, Hirata (1995) y Antunes (2002) afirman que en el escenario actual se observa una división sexual del trabajo que vincula las actividades de creación al trabajo masculino y las labores de menor calificación, frecuentemente basadas en una mayor explotación de la fuerza de trabajo, a las mujeres, inmigrantes y negros/as ${ }^{4}$. Esta nueva división sexual del trabajo -que se evidencia paralelamente a la extracción del valor del trabajo reproductivo ${ }^{5}$-, se asocia a la situación de mayor inestabilidad y precariedad de las mujeres trabajadoras en el mercado laboral.

Particularmente en América Latina, junto con la tendencia creciente de incorporación de las mujeres al mercado del trabajo, en las últimas décadas se observan significativas diferencias salariales entre los sexos, el aumento de la tasa de desempleo femenina, yla mayor participación de las mujeres en las ocupaciones informales, las cuales dan cuenta de la situación de mayor precariedad de la fuerza de trabajo femenina. A estos factores se suma, según Harvey (1998), el resurgimiento de pequeñas empresas y sistemas de trabajo artesanal y familiar que se posicionan como piezas relevantes del sistema de producción actual, establecen relaciones paternalistas entre hombres y mujeres, y facilitan una mayor explotación de estas últimas en el ámbito doméstico.

Laincorporación de la fuerza detrabajo femenina a los trabajos intensivos y tercerizados más precarios, la apropiación de la experiencia del trabajo reproductivo para la multiactividad, los menores salarios, la doble extracción del valor

En otros contextos históricos, las labores de menor calificación pueden ser realizadas por grupos sociales diferentes a los señalados por los/as investigadores/as.

El trabajo reproductivo hace referencia al trabajo doméstico y de cuidados que permite la sobrevivencia material de la fuerza de trabajo, y que es asumido como parte de los roles sociales de género asignados a las mujeres. 
del trabajo -productivo y reproductivo-, y la dinámica permanente de inclusión y expulsión del mercado de trabajo, entre otros factores, evidencian una mayor productividad de la fuerza de trabajo femenina. Dicha fuerza es generada por una explotación más intensa basada en la división sexual del trabajo, operada por las nuevas formas de organización productiva y de división internacional del trabajo (Federici 2013; Antunes 2002; Hirata 1995).

Diversas autoras (Carrasco, Borderías y Torns 2011; Benería 2011, entre otras) sostienen que en el contexto actual se evidencia un proceso de reestructuración del trabajo de reproducción, puesto que es la reproducción global del sistema y de los seres humanos la que está en crisis. Con la masiva incorporación de las mujeres al mercado laboral, el tiempo ocupado en el trabajo de cuidado material y relacional que habitualmente era desarrollado de forma gratuita, principalmente por las mujeres de la familia (madres, hijas, hermanas, abuelas) se reduce, y las labores domésticas deben ser resueltas con personas externas a la familia, generalmente, mujeres inmigrantes en el caso de los países europeos.

Como sostienen Carrasco, Borderías y Torns (2011), la crisis de los cuidados de los países del norte ha originado procesos migratorios de mujeres desde los países del sur hacia los países más ricos, quienes ofrecen trabajo de "cuidadoras". Paralelamente, como señala Benería (2011), en los países "en desarrollo" el neoliberalismo ha debilitado y desuniversalizado la protección social, lo que ha provocado la feminización de la migración internacional. Este proceso ha contribuido a la globalización de los cuidados y de la reproducción social, que Hoschschild denomina "las cadenas mundiales de afectos y asistencia" (Cit. en Carrasco, Borderías y Torns 2011: 55). Benería (2011) y Hochschild (Cit. en Carrasco et al. 2011), entre otras autoras, sostienen que la formación de estas cadenas globales de cuidados está agudizando las desigualdades entre las propias mujeres, fundamentalmente en razón de la clase y de la etnia/raza.

Según Ehrenreich y Hochschild (Cit. en Fumagalli 2010), en este contexto se cruzan dos fenómenos que se potencian mutuamente: la internacionalización de la fuerza de trabajo que genera la movilidad masiva de migrantes, y la emergencia de una demanda de trabajo de nuevas actividades que antes eran desarrolladas en el ámbito de la reproducción, y que ahora forman parte del proceso de acumulación y de producción. En esta misma dirección, Fumagalli (2010) señala que, al igual que otra serie de parejas antitéticas sobre las que se basaba la estructura productiva y social del fordismo (manual / intelectual, asalariado / autónomo, tiempo de vida / tiempo de trabajo), la distinción entre las categorías de producción y reproducción ha perdido relevancia, fundamentalmente porque las facultades afectivas, relacionales y comunicativas que las mujeres utilizan en la familia se han convertido en las nuevas variables del mundo laboral en el capitalismo actual.

\section{Apropiación de habilidades "femeninas" en las fábricas de la charla}

Los resultados del estudio muestran que las diversas funciones que cumplen los/as teleoperadores/as y los/as supervisores/as en las fábricas de la charla establecen diferencias en las habilidades y las capacidades necesarias para sus respectivos trabajos. Se observan 
habilidades comunes en el caso de los/as teleoperadores/as, pero también exigencias diversas dependiendo de las plataformas y las tareas específicas que éstos/as deben desarrollar.

En los trabajos vinculados a la atención directa de los clientes se exige a los/as teleoperadores/ as la capacidad de comprender las necesidades del usuario de los servicios, y de resolver los problemas identificados en el menor tiempo posible. En las plataformas de atención de reclamos los/as trabajadores/as sufren constantemente agresiones y malos tratos, lo que les obliga a utilizar las capacidades de contención de la agresividad, de mediación entre los requerimientos de los usuarios y las normativas empresariales, y de negociación en la resolución de conflictos. La empatía y la capacidad de contención emocional con los clientes son mencionadas como habilidades centrales, especialmente, para el trabajo en campañas de asistencia.

En las plataformas orientadas a las ventas y a la contención ${ }^{6}$ de los clientes en las empresas se utilizan fundamentalmente las habilidades para convencer y seducir. Los/as teleoperadores/as utilizan su propia experiencia como consumidores/as para enfatizar las características de los productos que los transforman en algo "deseable", e intentan establecer una relación de cercanía personal con los clientes que les facilite el logro de las ventas. La cordialidad, la simpatía, e incluso la coquetería son mencionadas como estrategias habituales para establecer en corto tiempo una relación distendida con los clientes:

El trabajo en las plataformas de contención se orienta a evitar que los clientes terminen sus contratos de servicios.
Tenemos que generar la instancia de tratar de hacer la conversación agradable con el cliente, porque toda negociación es fría, entonces uno obviamente tiene que romper esos esquemas con quien está hablando, ser simpática, dulce (Edith, teleoperadora Sermec).

En estos casos, descubrir los dispositivos que suscitan el deseo de consumo augura resultados positivos. En este sentido, los/as trabajadores/as que se desenvuelven personal y laboralmente en el contexto de una sociedad de masificación del consumo, la cual opera con la lógica del deseo, deben satisfacer dicho fin.

A nivel general, los/as trabajadores/as requieren ciertas habilidades comunes a todos los/as teleoperadores/as de call centers. Las capacidades de comunicación, el manejo a nivel de usuario de las plataformas computacionales y, especialmente, la "sonrisa telefónica", como símbolo de la obligatoria atención amable y cordial con los clientes, son mencionadas por la gran mayoría de los sujetos entrevistados como habilidades básicas para desempeñarse en las fábricas de la charla.

Muchas de las habilidades requeridas por los/ as teleoperadores/as de las fábricas de la charla -tales como la capacidad de convencimiento, la seducción, la mediación, la empatía, entre otras- son reconocidas socialmente como características asociadas al género femenino en las sociedades occidentales de las cuales forma parte Chile. Las capacidades necesarias para este trabajo, tanto a nivel general como en las plataformas específicas, son, en la mayoría de los casos, habilidades humanas que no requieren una calificación laboral especializada, y que forman parte de los procesos de socialización de las mujeres. 
De esta manera, las capacidades de comunicación, de comprensión, de resolución de problemas, de síntesis, de convencimiento, etc., son habilidades habitualmente utilizadas en los empleos vinculados al sector de servicios y también en el trabajo doméstico y de cuidados. La utilización masiva de la fuerza de trabajo femenina en los call centers -en Chile, así como en la mayoría de los países de América Latina con presencia de estas empresas- podría, entonces, vincularse con la explotación de estas habilidades reconocidas socialmente como "propiamente femeninas".

Según los antecedentes aportados por algunas trabajadoras entrevistadas, existiría una tendencia a que los teleoperadores varones se ocupen en las plataformas de servicio técnico, es decir, en una labor vinculada con la tecnología y que podría implicar un nivel mayor de complejidad. Sin embargo, no existen datos concretos que permitan afirmar esta tendencia, y en el caso de que fuese corroborada, es necesario considerar que el nivel de complejidad de este tipo de trabajo supera levemente el manejo de usuario/a.

La formación y la experiencia mínima requerida para trabajar en un call center, la escasa capacitación, la utilización de conocimientos técnicos básicos de manejo masivo, la explotación de capacidades humanas básicas y de habilidades reconocidas socialmente como "femeninas", dan cuenta de una "profesionalidad" que no requiere, como en el fordismo clásico, una formación específica adquirida en el trabajo, sino que se aprovechan las mismas habilidades usadas fuera del ámbito laboral. Estas constataciones plantean elementos de debate en dos aspectos relevantes de la discusión feminista sobre la división sexual del trabajo: 1) el carácter de los trabajos remunerados habitualmente asignados a las mujeres, y 2) las relaciones entre la producción y la reproducción y sus distinciones en el mundo dentro/fuera del trabajo.

En contraposición a las posturas que señalan que el trabajo de las mujeres es requerido en los empleos de menor calificación, Mazzei (2006) señala que ciertas labores que realizan las teleoperadoras brasileñas en los call centers -como manejar la información específica y cambiable de las campañas- son tareas que requieren destrezas de mayor complejidad. Si bien es posible reconocer algunas de estas habilidades complejas en los casos estudiados, especialmente en el manejo de la intensidad del trabajo, en general la mayoría son capacidades humanas subjetivas, muchas de ellas utilizadas por las mujeres en el ámbito doméstico.

Es, justamente, este carácter de habilidades formadas en los procesos de socialización de género el que parece no ser considerado en el debate de la corriente operaista italiana que propone la explotación de las habilidades humanas más básicas en el capitalismo cognitivo. Efectivamente, muchas de las habilidades apropiadas en los call centers son capacidades humanas básicas, pero forman parte de las asignaciones de género dominante en las sociedades occidentales.

Algunas habilidades complejas utilizadas como la polivalencia o el manejo de la intensidad laboral- forman parte, además, de los nuevos requerimientos exigidos a la fuerza de trabajo, especialmente en el sector de servicios, donde laboran masivamente las mujeres. La utilización intensiva del habla en los call centers, en cambio, podría ser considerada como la apropiación de una habilidad subjetiva y humana básica. Como señala Virno (Cit. en Colectivo Situaciones 
2006), el lenguaje incluido en el trabajo es una novedad absoluta en nuestra época, y emerge como una característica relevante del capitalismo contemporáneo.

La fuerza de trabajo de los call centers en Chile está compuesta mayoritariamente por mujeres teleoperadoras. Sin embargo, no se observan distinciones significativas en los requerimientos exigidos a los hombres o a las mujeres, ni tampoco diferencias salariales por sexo. A la minoritaria fuerza de trabajo masculina en los call centers le son requeridas habilidades socialmente definidas como "femeninas", lo que podría vincularse con la incorporación de personas con opciones homosexuales -especialmente gays-, quienes no son discriminados en este espacio laboral como ocurre en la gran mayoría de los trabajos en Chile. La constatación de la apropiación de ciertas habilidades requeridas por el capital, independientemente del sexo del trabajador/a, remite a las discusiones de las corrientes transfeministas (Solá y Urko 2013), y plantea la interrogante sobre los nuevos procesos de socialización de género en el capitalismo contemporáneo y sus mercados de trabajo.

El segundo eje de discusión planteado sostiene, con Fumagalli (2010) y Virno (Cit. en Colectivo Situaciones 2006), que la utilización de las mismas capacidades, tanto dentro como fuera del mundo laboral, podría generar una falta de distinción entre el tiempo de trabajo y el tiempo de la vida cotidiana. Lo anterior volvería obsoleta la distinción entre los espacios productivo y reproductivo.

Las capacidades requeridas por el capital en los call centers son capacidades humanas que se adquieren en los procesos de socialización, y son parte constitutiva de los seres humanos y de la vida misma. Sin embargo, la intensidad y la rígida organización del trabajo en que las facultades humanas básicas son explotadas, le otorgan un carácter distintivo a los call centers.

La explotación de las capacidades y las habilidades subjetivas en las fábricas del habla requiere de múltiples mecanismos de control y rutinización que operan en la organización taylorizada del trabajo en estas empresas. La medición del tiempo de conexión, el script o guión, el Tiempo Medio de Operación (TMO) ${ }^{7}$, el control de calidad, el logro de metas, el control de los/as supervisores/as y la disposición de los espacios, dan cuenta de la relevancia de la medición y del control estricto como factores centrales para disciplinar el habla y los cuerpos, y aumentar la productividad de los trabajadores/as. La repetición permanente de una labor específica en la cadena productiva en la rígida organización fordista del trabajo- se transforma en una repetición permanente en el uso del habla en los call centers.

En efecto, es la vida misma la que es puesta a trabajar en la fábrica de la charla, pero a un ritmo y bajo lógicas de control diferentes a las que operan en la vida cotidiana. Las habilidades subjetivas que durante el fordismo clásico eran usadas en la vida social y en la participación social, cultural y política, se convierten ahora en un factor productivo. De hecho, las distinciones entre el mundo dentro y fuera del trabajo se atenúan, pero mantienen ciertos rasgos diferenciadores en el caso de los call centers.

EI TMO mide los minutos exactos de duración de las llamadas que los/as teleoperadores/as realizan, y es una medición considerada en el control de calidad del trabajo, por tanto, relevante en la definición de la parte variable del salario de los/as teleoperadores/as. 
La utilización de las habilidades subjetivas especialmente del habla- en los call centers, que no requieren calificación sino la dada en la vida misma, implica que estas empresas no se caracterizan por la creación de conocimientos ni por la utilización de capacidades cognitivas complejas. Esta distinción podría matizar la noción de división cognitiva internacional de la producción propuesta por Fumagalli (2010), en la cual se produce una relación de interdependencia entre la producción de conocimiento y tecnologías de las grandes empresas del norte del mundo, y la actividad productiva de bienes materiales exigida a ejes internacionales de subcontratación.

La actividad de los call centers en Chile y en otros lugares de América Latina podría implicar que los países del capitalismo periférico no sólo actúan como productores de bienes, sino también como productores de riqueza a través de la utilización taylorizada de las habilidades subjetivas. Así como los países del capitalismo central explotan capacidades cognitivas que generan conocimientos nuevos que se expropian a través de la propiedad intelectual, en el caso de los call centers de los países del capitalismo periférico, se expropian las capacidades humanas "femeninas" a través de la taylorización del habla.

\section{Trabajo doméstico y crianza: Co-responsabilidad entre mujeres}

Las conexiones entre el espacio del trabajo y el espacio doméstico han sido reconocidas y analizadas largamente por la teoría feminista, más aún a partir de las evidencias de la doble explotación del trabajo de las mujeres en el capitalismo. Por ello, en este apartado se revisa la división sexual del trabajo que se evidencia en las familias de los/as trabajadores/as de call centers.

La mayoría de los/as trabajadores/as entrevistados/as tiene al menos un hijo/a y convive con los miembros de su familia nuclear. Considerando que la fuerza de trabajo de los call centers es fundamentalmente población joven, y que habitualmente en Chile los/as jóvenes se independizan tardíamente de su familia, es comprensible que la gran mayoría de los sujetos de la investigación constituya su unidad doméstica en convivencia con su familia de origen, habitualmente las madres, los padres, hermanos/as y abuelos/as.

Como se constata la siguiente entrevista, la convivencia con la familia de origen es un apoyo relevante para la vida cotidiana de los/as trabajadores/as, en cuanto permite compartir el trabajo doméstico, la crianza de los/as hijos/as, y también los costos de la economía doméstica:

cuando estoy trabajando mi abuela hace las cosas de la casa, yo solamente las de mi hijo, las colaciones, la ropa, cuadernos, todas esas cosas. Pero el día sábado y domingo me toca hacer de todo, lavar, planchar, aseo, cocinar (...) (Yeny, teleoperadora Atento).

En casi todos los casos, las mujeres asumen la crianza de los/as hijos/as con el apoyo de sus madres, abuelas y, en algunos casos, de hermanos/as mayores. Sólo en algunos casos de trabajadoras con una relación de convivencia en pareja, se observa la participación de los hombres en el trabajo doméstico y de cuidado de los/as hijos/as. Tanto en los casos de teleoperadoras con pareja estable o sin ella, se evidencia que los padres de los/as hijos/as de las trabajadoras tienen un rol mínimo o nulo 
tanto en el mantenimiento económico, como en el cuidado y la crianza de los/as niños/as.

El cumplimiento de los derechos maternales que se constata en las fábricas de la charla, a través de la financiación de salas cunas - cuidadoras para los/as menores, permite también un soporte en el cuidado de los/as hijos/as. A diferencia de muchas empresas en Chile que utilizan estrategias para evitar cumplir los derechos maternales, todas las trabajadoras entrevistadas señalan que en los call centers se respetan los periodos de pre y posnatal, y se financian los costos de salas cunas o cuidadoras para sus hijos/as.

Se observa, entonces, una co-responsabilidad en el trabajo y la economía doméstica, y también en la crianza de los/as hijos/as, lo que en estricto rigor no sitúa a las entrevistadas como "jefas de hogar", sino más bien como partes de una densa red de colaboración familiar entre mujeres, la cual se instala como un soporte importante ante los salarios variables de los/as trabajadores/as de call centers. La mayoría de las trabajadoras entrevistadas asume un papel relevante en la economía doméstica, pero su aporte varía dependiendo de sus ingresos.

Uno de los atractivos mencionados por las trabajadoras entrevistadas para buscar empleo y mantenerse en las empresas de call centers es la posibilidad de conciliar el trabajo doméstico con el trabajo remunerado. Las jornadas de trabajo parciales, la posibilidad de modificar el contrato laboral y disminuir la jornada de 45 horas semanales, la flexibilidad horaria en algunas empresas, son destacadas como factores fundamentales para conciliar el trabajo asalariado en las fábricas de la charla con el cuidado y la crianza de los/as hijos/as: por eso tengo este trabajo, porque por ejemplo cuando yo llego mis hijos están en clase me pongo a ordenar, ellos llegan, preparo once [comida de la tarde], les ayudo en las tareas. Igual alcanza el tiempo. Estuve unos meses haciendo promociones en supermercados y los turnos eran larguísimos, no pude seguir trabajando ahí (Marina, teleoperadora Sermec).

La constatación de que la gran mayoría de las trabajadoras entrevistadas realiza el trabajo asalariado en los call centers junto al trabajo doméstico y de cuidado, permite sostener que en las fábricas de la charla se realiza una doble extracción del valor del trabajo productivo y reproductivo de las mujeres trabajadoras. Sin embargo, la participación central de la familia en el trabajo reproductivo incorpora una nueva dimensión a la explotación de la fuerza de trabajo. En estos casos, el capital no sólo utiliza el trabajo reproductivo de las trabajadoras de los call centers, sino también el de otras mujeres ${ }^{8}$ que componen la unidad doméstica. La explotación de la fuerza de trabajo se extiende, por lo tanto, a la unidad doméstica a través de los roles de género socialmente asignados a las mujeres en las sociedades occidentales.

A diferencia del proceso de globalización de los cuidados, que ha permitido satisfacer en alguna medida las necesidades de trabajo doméstico y de cuidados de los países del norte a través de la migración de las mujeres de los países del sur (Carrasco, Borderías y Torns 2011; Benería 2011; Hoschschild Cit. en Carrasco, Borderías y Torns 2011; Federici Cit. en Fumagalli 2010, entre otras), el caso de los call centers en Chile indica que la responsabilidad y la ejecución

Si bien en algunos casos se menciona el trabajo de abuelos y hermanos mayores de las ejecutivas de call centers en el trabajo doméstico y de cuidado y crianza de los/as hijos/as, habitualmente lo realizan las madres y las abuelas de éstas. 
del trabajo reproductivo y de cuidado continúa siendo de las mujeres, tanto de las que laboran en el ámbito productivo, como de las que trabajan en el ámbito doméstico no remunerado.

Como sostiene Benería (2011), las mujeres de clases media y alta en América Latina pueden acceder al trabajo doméstico remunerado. Sin embargo, no es el caso de las trabajadoras de las fábricas de la charla. Los salarios de estas trabajadoras no les permiten acceder al trabajo remunerado de otras mujeres para desarrollar el trabajo doméstico, y el desmantelamiento del Estado del Bienestar tampoco les permite acceder a servicios públicos de calidad.

La utilización del trabajo doméstico y de cuidado no remunerado de las mujeres de la red familiar de las trabajadoras de call centers implica, por una parte, que efectivamente se recurre al trabajo de otras mujeres, pero en una lógica diferente a la observada en los países del norte, en cuanto no se accede al trabajo doméstico ofrecido en el mercado, sino al trabajo no remunerado disponible en el espacio doméstico. Por otra parte, esta situación implica que el salario de la teleoperadora sigue dando cuenta tanto del trabajo productivo como del trabajo reproductivo, pero no sólo de la trabajadora del call center, sino también de otras mujeres de la red familiar.

A diferencia del patrón clásico en que las mujeres realizan el trabajo doméstico que permite reproducir la fuerza de trabajo masculina, en el caso de las fábricas de la charla son las propias mujeres las que permiten la reproducción de la fuerza de trabajo femenina. Si bien algunas teleoperadoras entrevistadas reconocen en su discurso la legitimidad de la participación de los hombres en el trabajo doméstico y de cuidado, en la práctica concreta la responsabilidad y la ejecución del trabajo reproductivo sigue siendo de las mujeres -trabajadoras y familiares-, lo que permite afirmar que la división sexual del trabajo se mantiene.

Por último conviene destacar que, a pesar de que algunas investigaciones sostienen que los sectores juveniles en Chile son más proclives a realizar cambios en los roles socialmente asignados a hombres y mujeres (INJUV 2010; Gutiérrez y Osorio 2008), en el caso de los/as trabajadores/as de call centers que forman parte de una fuerza de trabajo juvenil no se observa esta tendencia. Posiblemente, esto se deba a su convivencia con las familias nucleares, en la mayoría de los casos.

\section{Reflexiones finales}

Los antecedentes analizados muestran que en la organización interna del trabajo en los call centers no se evidencia una división relevante en la asignación de tareas por sexo. Tampoco se evidencian diferencias salariales entre hombres y mujeres, ni desigualdades en las posibilidades de ascenso o de movilidad al interior de las empresas. La lógica de división sexual del trabajo asalariado opera a través de la explotación intensiva de las capacidades humanas subjetivas reconocidas socialmente como "femeninas", y que podrían explicar, en parte, la mayoritaria incorporación de las mujeres en estas empresas. A su vez, la división sexual del trabajo por ramas de actividad es una tendencia extendida en el mundo laboral chileno, donde la fuerza de trabajo femenina se concentra en los sectores ligados a la prestación de servicios y al trabajo de cuidados, labores vinculadas a los roles socialmente asignados a las mujeres. 
En las entrevistas realizadas se constata que en el ámbito doméstico se mantiene la división sexual del trabajo en las responsabilidades por el trabajo doméstico y de cuidado de los/ as hijos/as asumidas por las mujeres. La doble extracción del trabajo productivo y reproductivo de las trabajadoras de call centers presenta la particularidad de incorporar a otras mujeres de la red familiar en la realización del trabajo en el ámbito doméstico.

La explotación en los call centers de las capacidades humanas subjetivas consideradas socialmente como atributos "femeninos" hacen que las labores que se realizan dentro y fuera del trabajo sean similares. Las habilidades empleadas en el trabajo reproductivo y de cuidado -empatía, negociación, afectos, entre otras- son utilizadas también en el espacio productivo de las fábricas de la charla.

El rol de las mujeres de la red familiar de las trabajadoras en el desarrollo de la actividad laboral asalariada, sumado a la constatación de la similitud de habilidades utilizadas en los trabajos productivo y reproductivo, permiten sostener, en este caso, que la dicotomía que separaba el espacio dentro/fuera del trabajo remunerado que se estableció durante el fordismo clásico se desdibuja (Fumagalli 2010).

En este estudio se evidencia cómo los roles de género -que asignan a las mujeres el trabajo doméstico y de cuidado y que les atribuyen cualidades subjetivas particulares-, siguen siendo utilizados en los nuevos formatos de organización de la producción y del trabajo en el capitalismo contemporáneo. En efecto, como sostiene Stolcke (1988), las transformaciones en las relaciones productivas han sido mediadas por valores culturales de género, y por la división sexual del trabajo que ha resultado de ellas. La doble extracción del valor del trabajo productivo y reproductivo de las trabajadoras y las mujeres de su familia, y el aprovechamiento de las cualidades socialmente reconocidas como "femeninas", dan cuenta de una mayor explotación de las mujeres trabajadoras chilenas en las fábricas de la charla.

La incorporación de las mujeres en el mercado de trabajo ha aumentado en Chile en los últimos años. Sin embargo, el caso de los call centers demuestra que las trabajadoras no acceden al trabajo doméstico y de cuidados remunerado, sino al de las mujeres de su red familiar. Si la dinámica que ocurre en las fábricas de la charla se reproduce en otros sectores laborales, es posible suponer que la mayor participación de las mujeres en el mundo del trabajo en el escenario del desmantelamiento del Estado del Bienestar ha implicado la intensificación de la explotación de las mujeres, tanto de aquellas que están dentro, como de las que están "aparentemente" fuera de la esfera laboral asalariada. Esto pone en cuestión, como señala Federici (2013), la incorporación al trabajo asalariado como camino de liberación de las mujeres.

A la utilización de una fuerza de trabajo más barata en los países del capitalismo periférico, y de las políticas estatales de atracción de inversiones extranjeras por los países llamados "subdesarrollados", se suma la explotación de las capacidades y las habilidades subjetivas humanas, especialmente aquellas reconocidas como "femeninas". Las empresas de call centers muestran una gran agilidad para movilizar sus capitales a nivel mundial y nacional -como se evidencia en la creciente migración de call centers a países de América Latina con menores costos laborales-, además, 
explotan intensivamente la fuerza de trabajo a través del taylorismo telefónico, se apropian del trabajo productivo y reproductivo de una fuerza de trabajo femenina masiva y de las mujeres de su red familiar, y utilizan los roles y los valores culturales asociados con lo "femenino".

\section{Bibliografía}

Antunes, R. 2002. Os sentidos do trabalho. Ensaio sobre a afirmaçâo e a negaçâo do trabalho. Sâo Paulo: Editorial Boitempo.

Baeza, M. 2002. De las metodologías cualitativas en la investigación científico-social. Diseño y uso de instrumentos en la producción de sentido. Concepción: Universidad de Concepción.

Benería, L. 2011. "Crisis de los cuidados. Migración internacional y políticas públicas". El trabajo de cuidados. Historia, teoría y políticas. Carrasco, C., Borderías, C. y Torns, T. (Eds.). Madrid: Los Libros de la Catarata. 359389.

y Roldán, M. 1992. Las encrucijadas de clase y género: Trabajo a domicilio, subcontratación y dinámica de la unidad doméstica en la ciudad de México. México D.F.: Fondo de Cultura Económica.

Carrasco, C., Borderías, C. y Torns, T. 2011. "El trabajo de cuidados: Antecedentes históricos y debates actuales". El trabajo de cuidados. Historia, teoría y políticas. Carrasco, C., Borderías C. y Torns, T. (Eds.). Madrid: Los Libros de la Catarata. 13-96.

Centro de Estudios Nacionales de Desarrollo Alternativo y Fundación Instituto de Estudios Laborales. 2010. Derechos en el trabajo y relación de equidad de género en el mercado laboral: El caso del call center. Santiago: CENDA-FIEL.

Colectivo Situaciones. 2006. ¿Quién habla? Lucha contra la esclavitud del alma en los call centers. Buenos Aires: Ediciones Tinta Limón.

Del Bono, A. 2010. "La geografía de los call centers: Territorio, trabajo y empleo". La llamada... el trabajo y los trabajadores de call centers en Córdoba. Roitman, S. Lisdero, P. y Marengo, L. (Comps.). Buenos Aires: Jorge Sarmiento Editor-Universitas Libros. 37-66.

Federici, S. 2013. Revolución en punto cero. Trabajo doméstico, reproducción y luchas feministas. Madrid: Traficantes de Sueños.

Fumagalli, A. 2010. Bioeconomía y capitalismo cognitivo. Hacia un nuevo paradigma de acumulación.
Madrid: Traficantes de Sueños.

Gutiérrez, E. y Osorio, P. 2008. "Modernización y transformaciones de las familias como procesos del condicionamiento social de dos generaciones". Revista Última Década 29: 103-135.

Harvey, D. 1998. La condición de la posmodernidad. Investigación sobre los orígenes del cambio cultural. Buenos Aires: Amorrortu Editores.

Hirata, H. 1995. "Rapports sociaux de sexe et division du travail: Contribution à la discussion sur le concept de travail". La crise du travail. Actuel Marx Confrontation. Bidet, J. y Texier, J. (Eds.). París: Press Universitaires de France. 81-92.

Instituto Nacional de la Juventud. 2010. Sexta Encuesta Nacional de Juventud. Santiago: INJUV.

Kremerman, M. 2005. En la ruta del trabajo decente: Análisis de los sectores salmonicultura, call center $y$ agroexportación. Santiago: Terram Publicaciones.

Mazzei, C. 2006. O trabalho duplicado. A divisão sexual no trabalho e na reprodução um estudo das trabalhadoras do telemarketing. São Paulo: Editora Expressão Popular.

Micheli, J. 2007. "Los call centers y los nuevos trabajos del siglo XXI". Revista CONfines 5: 49-58.

Molina, K. y Ruminot, N. 2010. La investigación acción participativa como modelo de investigación en los trabajadores de call centers de Chile. El caso de los trabajadores de Multivoice. Santiago: Instituto de Ciencias Sociales Alejandro Lipschutz.

Solá, M. y Urko, E. (Comps.). 2013. Transfeminismos. Episteme, fricciones y flujos. País Vasco: Txalaparta.

Stolcke, V. 1988. Coffee Planters, Workers \& Wives. Class Conflict and Gender Relations on Sao Paulo Plantations, 1850-1980. New York: St. Martin's Press.

Taylor, S. y Bogdan, R. 1986. Introducción a los métodos cualitativos de investigación. La búsqueda de significados. Barcelona: Editorial Paidós.

Uribe, V. y Morales, G. 2010. Atendiendo a los clientes de los clientes. La industria del Call Centers y sus condiciones laborales. Santiago: Dirección del Trabajo. 
